\title{
Measuring of filtration efficiency of nonwoven textiles in volume from scattered light by seeding particles
}

\author{
P. Bilek ${ }^{1}$ and P. Sidlof ${ }^{2}$ \\ ${ }^{1}$ Technical University of Liberec, Halkova 917/6, 46001 Liberec 1, Czech Republic, petr.bilek@tul.cz \\ ${ }^{2}$ Technical University of Liberec, Halkova 917/6, 46001 Liberec 1, Czech Republic, petr.sidlof @tul.cz
}

\begin{abstract}
This paper deals with the method which calculates a filtration efficiency of nonwoven textiles from scattered light intensity by seeding particles. The filtration efficiency is commonly measured by particle counters. Samples of liquid or gas are taken during a test in front of and behind a filtration material. The concentration of particles is measured and the filtration efficiency is calculated. The filtration efficiency does not have to be uniform in its whole surface. The uniformity of filtration is another indicator of a quality of filtration materials. Measurements described in this article were performed on a water filtration setup which enables optical access to the place where the filtration material is mounted. Pictures of illuminated seeding particles are made by a laser sheet and a camera. Visualisation of the filtration process enables measuring of the efficiency of separation versus time and also versus two-dimensional position in case of use of a traverse mechanism. The filtration textiles were tested by $1 \mu \mathrm{m}$ seeding particles. Mean value of light intensity and number of bright pixels in evaluative areas during image analysis were obtained. On the basis of these data, the filtration efficiency is calculated. The best image analysis method was chosen.
\end{abstract}

\section{Introduction}

\subsection{Investigation of filtration materials}

A filtration material represents an important element in many industrial processes. The filtration material is a barrier which retains suspended particles in a medium. The typical flat filtration material is a screen where the particles bigger then pores are collected on the surface. Pores and fibres have the same size in this case. By contrast, nonwoven materials are made from random deposited fibres. Pores have not the same size and the layer has not totally uniform thickness. The uniformity or homogeneity in thickness and the uniformity of fibres arrangement of a filtration material is an important quality. Filtration properties are influenced by the uniformity and homogeneity. The other filtration properties of the filtration textiles are permeability, pressure drop, filtration efficiency and environmental resistance. The permeability is directly proportional to the flow rate, fluid velocity, fluid viscosity and filter thickness and inversely proportional to the filter area and fluid density. The pressure drop $\Delta p$ is a difference between pressures in front of the filter $\mathrm{p}_{1}$ and behind it $\mathrm{p}_{2}$ (1) during constant the flow rate. The filtration efficiency $\mathrm{EF}$ is a ratio between concentrations upstream $\mathrm{G}_{1}$ and a downstream $G_{2}$ (2). All these parameters determine the total quality of the filtration materials [1].

$$
\begin{gathered}
\Delta p=p_{1}-p_{2}[P a], \\
E F=\left(1-\frac{G_{2}}{G_{1}}\right) \cdot 100[\%] .
\end{gathered}
$$

Quality of nonwoven materials can be investigated by offline methods under a focal or an electron microscope. Pictures from the microscope are image analysed. The method is very accurate but also time consuming. In addition, only a small sample of the filtration textile is investigated. Online research methods which are based on automated fibre sizing use the special software [2]. These methods do not describe behaviour of a filter during a process. The filtration efficiency can bemeasured online by artificial particles spread in a medium. The measuring is done on the basis of particle concentration using particle counter.

Standard methods of measuring of the filtration efficiency measure only the overall filtration efficiency versus time. In the present paper, a measuring method for diagnosis of a quality of the filtration materialsis described. Homogeneity of the filtration (the filtration efficiency versus two-dimensional position) is measured. 


\subsection{Visualization and light scattering}

In this article, the filtration efficiency is measured on the basis of visualization of a filtration process. The laser visualization of flow is well known e.g. from the Particle Image Velocimetry method (PIV), where a vector field of velocities is also diagnosed. A medium is seeded up by traced artificial particles. The flow is illuminated by a laser sheet. A camera records the whole illuminated scene during the filtration process.

The laser light is scattered by single particles and they become visible. Only an illuminated fog is observable in case of use of very small seeding particles. The more particles are in the medium, the more light is scattered. Number of detected particles n, light intensity I and number of bright pixels $\mathrm{Y}$ in the pictures from the camera is directly dependent on a concentration of seeding particles in water $G(3)$.

$$
n, I, Y \sim G
$$

The whole scattered light intensity unfortunately does not depend only on the concentration of the particles in a medium. The light intensity of a single particle is the function $\mathrm{I}_{\mathrm{sc}}=\mathrm{I}_{\mathrm{in}}\left(\theta, \lambda_{\mathrm{in}}, \varnothing, \mathrm{n}_{0}\right)$, where $\mathrm{I}_{\mathrm{in}}\left[\mathrm{W} / \mathrm{m}^{2}\right]$ is the intensity of a light source, $\theta\left[{ }^{\circ}\right]$ is the angle between the light source and a detector, $\lambda_{\text {in }}[\mathrm{m}]$ is the wavelength of an incident light, $\varnothing[\mathrm{m}]$ is the diameter of the particle and $\mathrm{n}_{0}[-]$ is therelative refractive index [3]. More theories describe the scattering phenomena. The basic approaches are divided according to parameters $\mathrm{x}$ and $\mathrm{n}_{0}(4)$.

$$
x=\frac{2 \pi \cdot \phi}{\lambda_{\text {in }}}[-], \quad n_{0}=\frac{n_{\text {particle }}}{n_{\text {medium }}}[-] .
$$

Mie theory has not any limitations on the other hand, but it does not exist in an analytical representation. Fortunately, many programs are written to help to solve this problem. The biggest problem in this way seems to be a description of a surface of the particles and ensuring perfectly spherical shape and smooth surface of them. Due to this, calculation of the scattered light intensity of non-spherical particles is not very precise.

Energy transported by electromagnetic waves is described by radiometric or photometric quantities. The radiometric quantities describe the energy in whole spectrum of frequencies. On the other hand the photometric quantities describe only the effect on a human eye. Light is measured as the radiometric quantity (radiance $\mathrm{E}_{\mathrm{e}}\left[\mathrm{W} / \mathrm{m}^{2}\right]$ or irradiance $\mathrm{L}_{\mathrm{e}}\left[\mathrm{W} / \mathrm{sr} \cdot \mathrm{m}^{2}\right]$ ). The radiometric quantity is recalculated to the range of human view according to the spectral sensitivity of a human eye. The results are photometric quantities illuminance $E[\mathrm{~lx}]$ and luminance $\mathrm{L}\left[\mathrm{cd} / \mathrm{m}^{2}\right]$. The luminance (light intensity) can be measured by a digital camera with CCD or CMOS chip $[4,5]$.

\section{Water-filtration setup}

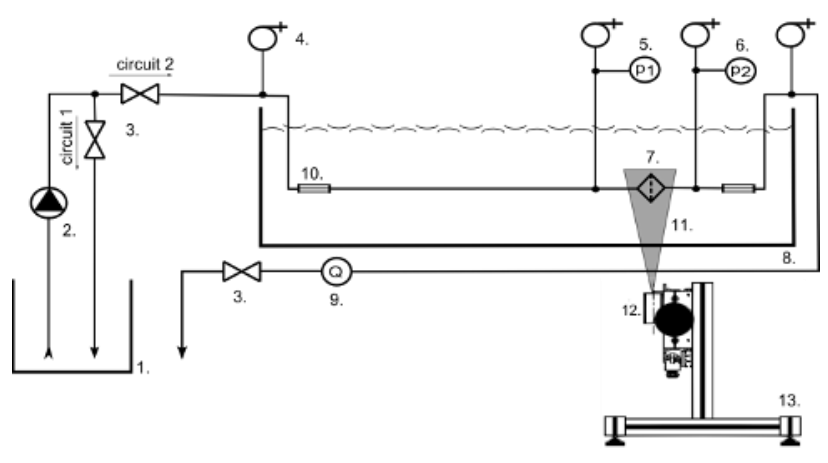

Figure 1. Scheme of the water-filtration setup (1: Reserve tank for water, 2: Controlled pump, Ondina $50 \mathrm{~T}, \mathrm{P}=0.37 \mathrm{~kW}$,

3: Manual ball valve, 4: Automatic air-escape valve, 5: Pressure sensor GMSD 3.5 BRE, Greisinger, 6: Pressure sensor GMSD 350 MRE, Greisinger, 7: Sample of a filtration material, $8 \mathrm{~cm}$ in diameter, 8: Glass tank for water, 9: Inductive flow meter

$\mathrm{M} 1500, \mathrm{Q}_{\max }=10$ liters per minute, Malema sensors, 10: Honeycomb screens, 11: Laser sheet, 12: Head with the laser unit, $\mathrm{P}=50 \mathrm{~mW}, \lambda=532 \mathrm{~nm}, 13$ : One axis traverse mechanism).
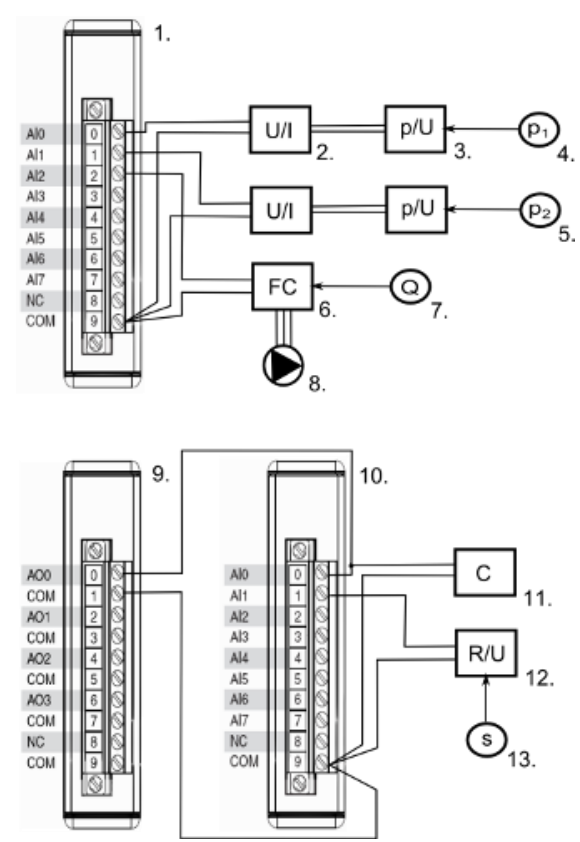

Figure 2. Electric wiring scheme of the water-filtration setup (1: National Instruments data acquisition module- 9203, 2: Converter Voltage - Current, DT 1100, 3: Measuring unit GMH 3161, 4: Pressure sensor GMSD 3.5 BRE, 0 - $350 \mathrm{kPa}$, 5: Pressure sensor GMSD $350 \mathrm{MRE}, 0-35 \mathrm{kPa}, 6$ : Frequency converter Siemens Micromaster 420, 7: Inductive flow meter M1500, Malema sensors, 8: Controlled pump, Ondina $50 \mathrm{~T}$, $\mathrm{P}=0.37 \mathrm{~kW}, 9$ : National Instruments voltage output module 9263, 10: National Instruments data acquisition module - 9201, 11: Camera Pike F-210B/C, 12: Converter Resistance - Voltage, 13: Position sensor of ahead of the traverse mechanism).

The water-filtration setup was built according to scheme in figure 1. Water with seeding particles is pumped by a controlled pump into the setup. Circuit 1 is used to ensure homogeneity of seeding particles in water in a reserve 
tank. Circuit 2 is used for measuring. The glass part of the setup with a sample of filtration material is immersed in a glass tank full of water to prevent optical distortion. Automatic air-escape valves are mounted on every high place in the circuit 2. The pressure drop is measured online by two sensors. The visualisation of the filtration process is ensured by laser sheet, seeding particles and black and white high resolution camera with CCD chip $1920 \times 1080 \mathrm{px}$. The filtration efficiency is optically measured on the basis of image analysis of 16 bit depth RAW format pictures from the camera.

The flow rate is stationary and is controlled by a frequency converter. Triggering signal for the camera is generated by NI voltage output module. Signals from the sensors are collected by NI data acquisition cards. All modules are plugged in the NI CompactDAQ - 9172 system which is connected with PC, see figure 2.

Software MACA (Measuring And Control Application) was written in LabView 2011 for data acquisition and measurement control in the water-filtration setup. This software controls the traverse mechanism movement, generates the trigger signal for the camera and processes signals from the data acquisition modules.

\section{Experiments}

Seeding particles and microfibrous textiles with details in table 1 and 2 were used. The sample of the filtration material was made from MB30 + $2 \times$ MB35 and laboratory called Sample100. Filtered clear tap water was used for experiments. Settings of the camera were set up according to table 3 . All settings during a calibration must be strictly the same as during the following measurements.

Table 1. Properties of the filtration materials.

\begin{tabular}{|l|l|l|}
\hline Laboratory name & MB 30 & MB 35 \\
\hline Surface weight & $30 \mathrm{~g} / \mathrm{m}^{2}$ & $35 \mathrm{~g} / \mathrm{m}^{2}$ \\
\hline Technology & meltblown & meltblown \\
\hline Material & polypropylen & polypropylen \\
\hline Diameter of fibers & $2-5 \mu \mathrm{m}$ & $2-5 \mu \mathrm{m}$ \\
\hline Structure & raster calander & free \\
\hline
\end{tabular}

Table 2. Properties of the seeding particles.

\begin{tabular}{|l|l|}
\hline Mark & Glass tripol D \\
\hline Main diameter & $1.75 \mu \mathrm{m}$ \\
\hline Dispersion & $1-5 \mu \mathrm{m}$ \\
\hline Density & $2.55 \mathrm{~g} / \mathrm{m}^{3}$ \\
\hline Shape & nonspherical \\
\hline Material & $\mathrm{SiO}_{2}$ \\
\hline
\end{tabular}

Table 3. Settings of the camera.

\begin{tabular}{|l||l|}
\hline Aperture & F1,8 \\
\hline Shutter & $15 \mathrm{~ms}$ \\
\hline Gain (amplification) & $500[-]$ \\
\hline Brightness & $0[-]$ \\
\hline Grey level & $125 \mathrm{bit}$ \\
\hline Bit depth & 16 bit \\
\hline
\end{tabular}

\subsection{Calibration of the filtration setup}

The aim of the first experiment was to measure a concentration of the seeding particles in water. It was done without any sample of filtration material. The laser sheet is directed into the middle of the glass pipe. The concentration of the artificial particles in the setup was increased step by step from zero to maximum. Five pictures of the illuminated seeding particles were madefor the each concentrationto ensure better accuracy.

\subsubsection{Image analysis of the made pictures}

Image analysis was done inthe software DOFES (Determination Of Filtration Efficiency Software). DOFES was written for this purpose in Matlab 2011. Settings of DOFES software are described in table 4. In figure 3 a sketch of the filtration process with evaluative areas for the image analysisis shown. One evaluative area is sufficient enough for the calibration measurement.

Table 4. Settings of DOFES software.

\begin{tabular}{|l|l|}
\hline Number of couples of areas & 11 \\
\hline Size of evaluation areas & $\mathbf{S}_{\text {area }}=80 \times 80 \mathrm{px}$ \\
\hline Minimum grey level & $\mathbf{y}_{\text {min }}=2432$ \\
\hline Maximum grey level & $\mathbf{y}_{\text {max }}=65535$ \\
\hline Step in histogram & 128 \\
\hline
\end{tabular}

The simplest way of the image analysis is to detect a number of seeding particles in an evaluative area. But the particles must be visible enough, and distinguishable from each other. Concentration of the particles must be in suitable bounds. The evaluative areas must be sufficiently large. This method is suitable only in case of use of particles bigger than approximately $5 \mu \mathrm{m}$ due to a good visibility.

The second method is a calculation of mean digital grey value. There are no limitations in a value of concentrations of the seeding particles. But this method is very sensible on impurities and light reflections. A bigger and well illuminated particle can depreciate the result of the whole evaluative area. Therefore, the calculation must be done in defined bounds of digital grey value. A curve of concentration of particles versus mean digital grey value is shown in figure 4 . 


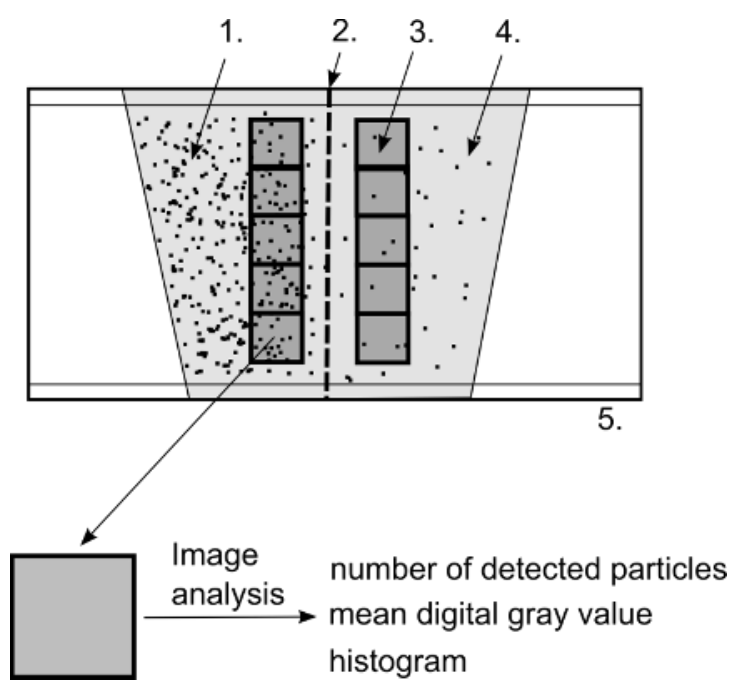

Figure 3. Image analysis of the pictures from the camera (1: Illuminated seeding particles in front of a filter, 2: Sample of a filtration textile, 3: Evaluative area for image analysis,

4: Illuminated seeding particles behind the filtration media, 5: Glass pipe.).

The last method is a creation of a histogram of the seeding particles in defined bounds of the digital grey value. Figure 5 demonstrates that the relation between the concentration of the particles and the number of the bright pixels is linear with better R-squared value than in the previous case. This method seems to be the most suitable for our experiments.

$$
n=\frac{1000000 \cdot \sum_{y_{\min }}^{y_{\max }} n_{h}}{S_{\text {area }}}[-/ M p x]
$$

Pixels in an evaluative area, which have digital grey value between minimum $\mathrm{y}_{\min }[-]$ and maximum $\mathrm{y}_{\max }[-]$ values are summed. The digital grey values $y_{\min }[-]$ and $y_{\max }[-]$ are experimentally determined according to the histogram, see figure 6 . The sum of the pixels from the histogram $n_{h}[-]$ is normalized by the surface of the evaluative area $S_{\text {area }}[p x]$ and expressed in Mpx (5). The result is a number of bright pixels per $\mathrm{Mpx} n[-/ \mathrm{Mpx}]$.

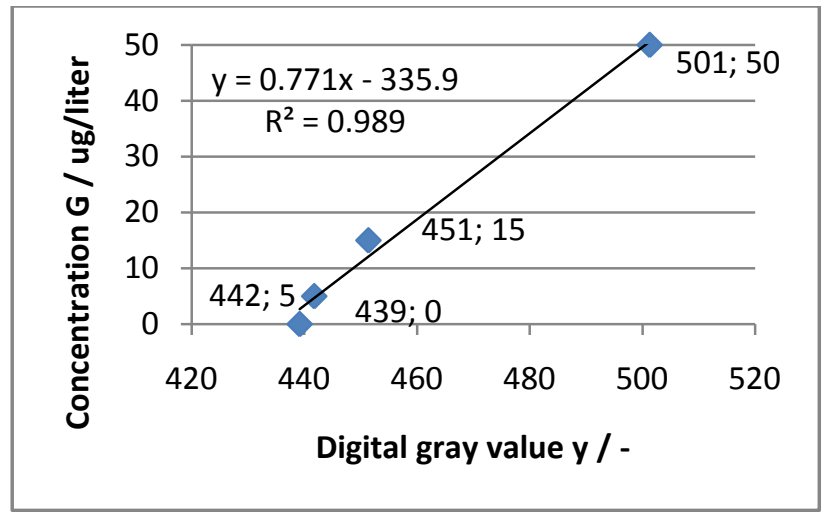

Figure 4. Graph shows a concentration of the particles in water versus mean digital grey value.

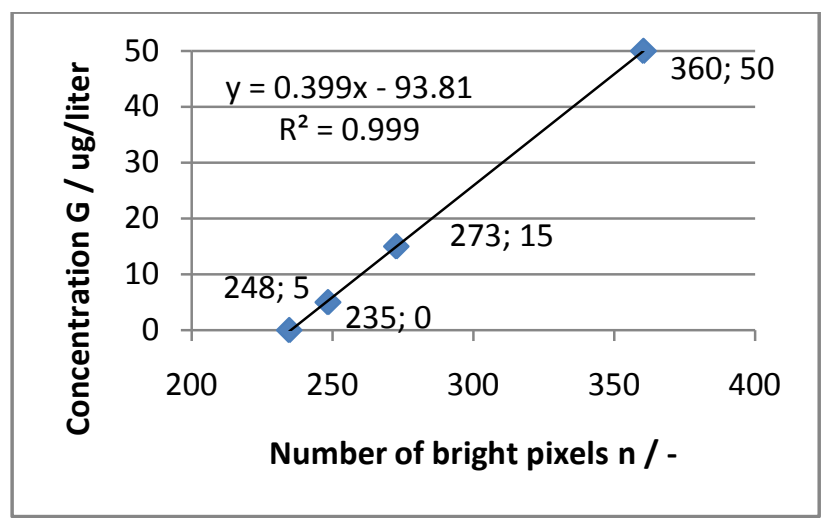

Figure 5. Graph shows a concentration of the particles in water versus number of bright pixels/Mpx.

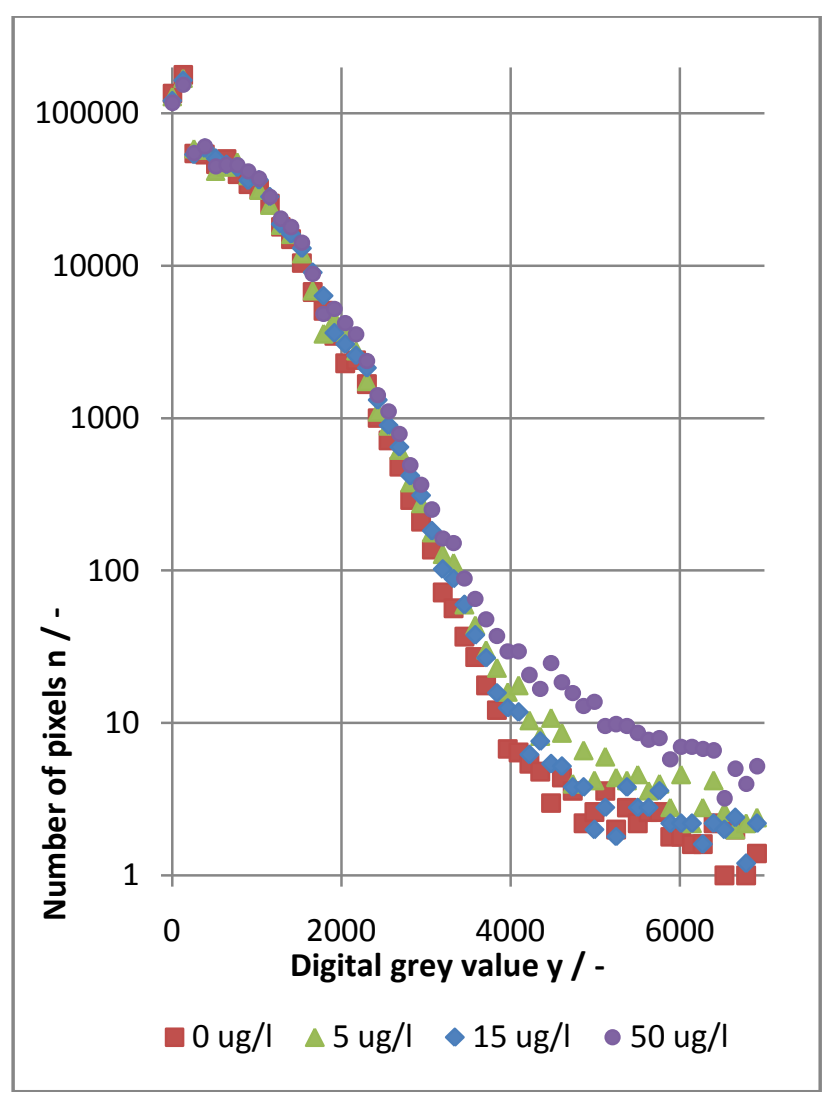

Figure 6. Graph shows the histogram of the number of pixels versus digital grey value.

\subsection{Measurement of the filtration efficiency in 3D}

Sample100 was mounted together with a protective spunbond textile, a supporting brass grid and seals between two glass cylinder pipes. Flow rate was adjusted to approximately 1 liter/minute by a manual ball valve and then controlled automatically by the frequency converter with implemented $\mathrm{PI}$ regulator $(\mathrm{P}=0.65[-]$ and $\mathrm{I}=1.8 \mathrm{~s})$. Settings of MACA software are shown in table 5. Sample 100 was tested by suspension with a concentration $500 \mu \mathrm{g} / 1$. 
Table 5. Settings of MACA software.

\begin{tabular}{|l||l|}
\hline Sample rate & $1 \mathrm{kHz}$ \\
\hline Decimation & $100[-]$ \\
\hline Moving average & 1000 samples \\
\hline Number of pictures & $11[-]$ \\
\hline Time of movement & $6 \mathrm{~s}$ \\
\hline Time of whole cycle & $30 \mathrm{~s}$ \\
\hline
\end{tabular}

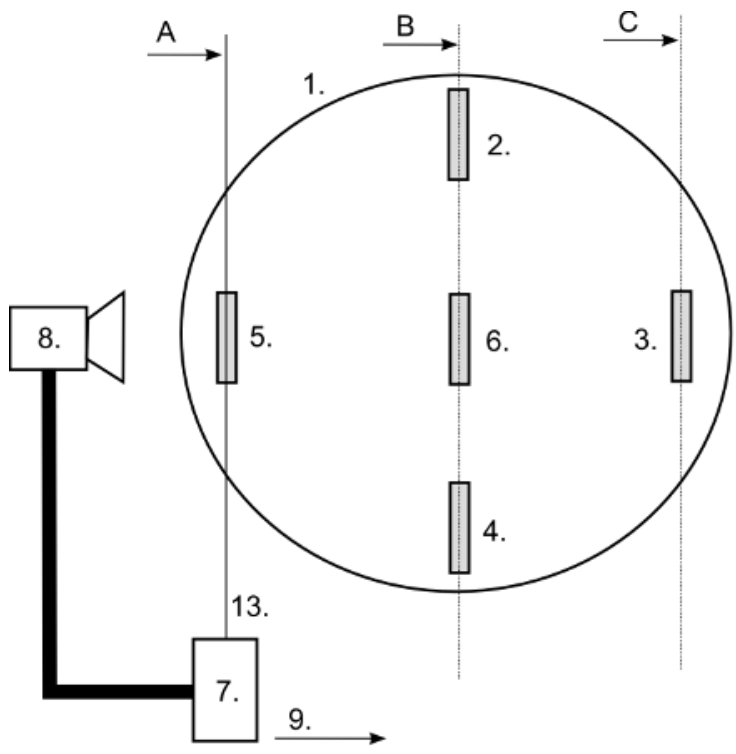

A, C:

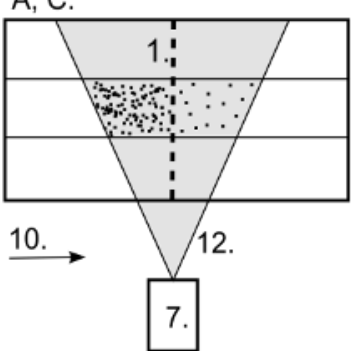

B:

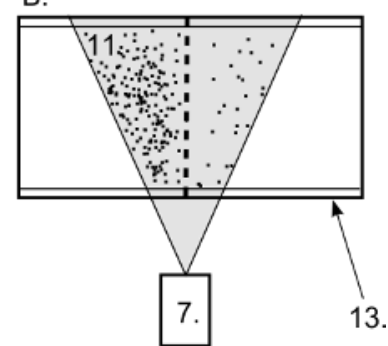

Figure 7. Sketch of an arrangement of the experiment

(1: Sample of the filtration textile, 2: Upper area, where was measured the filtration efficiency versus time, 3 : Right area,

4: Lower area, 5: Left area, 6: Middle area, 7: Laser unit, 8: Camera, 9: Movement of the camera and the laser unit by the traverse mechanism, 10: flow direction, 11: Illuminated seeding particles in front of the filter, 12: Laser sheet, 13: Glass cylinder pipe.).

Eleven images of the filtration process were recorded during every movement of the traverse mechanism. The traverse movement of the laser head took 6 seconds. The laser sheet is transferred from position A to position $\mathrm{C}$ or vice versa. The movement is performed every 30 seconds.

Figure 7 shows three basic positions of the camera and the laser unit (positions A, B and C). The camera and the laser unit are mechanically mounted to the head of a uniaxial traverse mechanism. At the beginning of measurement, the filtration efficiency can be calculated only from narrow illuminated strip in position A. The illuminated strip becomes wider during movement of the head of the traverse mechanism (with the laser unit and the camera) to the centre of the pipe to the position B. The illuminated area is the widest in the middle of the pipe in the position B. The illuminated area is again narrower in the direction of the position $\mathrm{C}$.

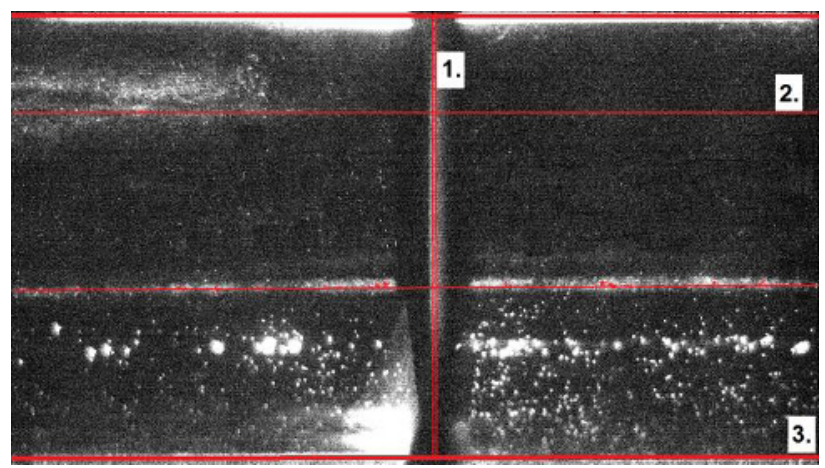

Figure 8. Typical picture of the filtration process in a backstop (position A or C) from the camera with highlighted (1: Sample of a filtration textile, 2: Narrow illuminated strip of seeding particles, 3: Edge of the glass pipe.).

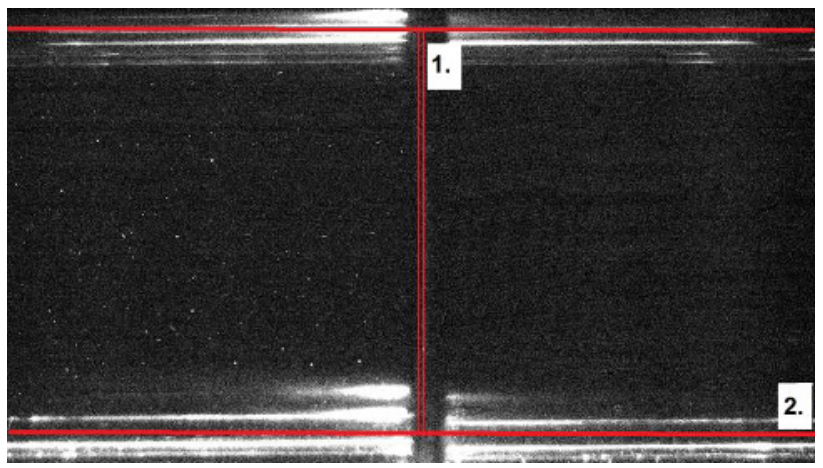

Figure 9. Typical picture of the filtration process in the middle of a glass pipe (position B) with highlighted (1: A sample of a filtration textile, 2: Edge of glass pipe.).

Figures 8 and 9 show the real filtration process. Artificial seeding particles are caught up on the front side of the filtration textile. Flow direction is from left to right.

\subsection{Results of the experiments}

The calibration curve was measured. The curve is approximately linear only in case of small concentrations. If the concentration of particles is high a multiscattering by seeding particles is significant. The maximum concentration $100 \mu \mathrm{g} /$ liter can be approximately used.

The filtration efficiency was calculated in five places of the sample, see figure 10 . The filtration efficiencies versus time in left, upper and middle area of the sample have similar values. The filtration efficiencies versus time in right and lower area of the sample are quite different. This can be caused by more factors.

One of them is light reflection of the laser light from mechanical parts and bubbles. This method is very 
sensitive to light reflections. On figure 11, an excessive light reflection which can degrade results is shown. The light reflection is the biggest in proximity of mechanical parts.

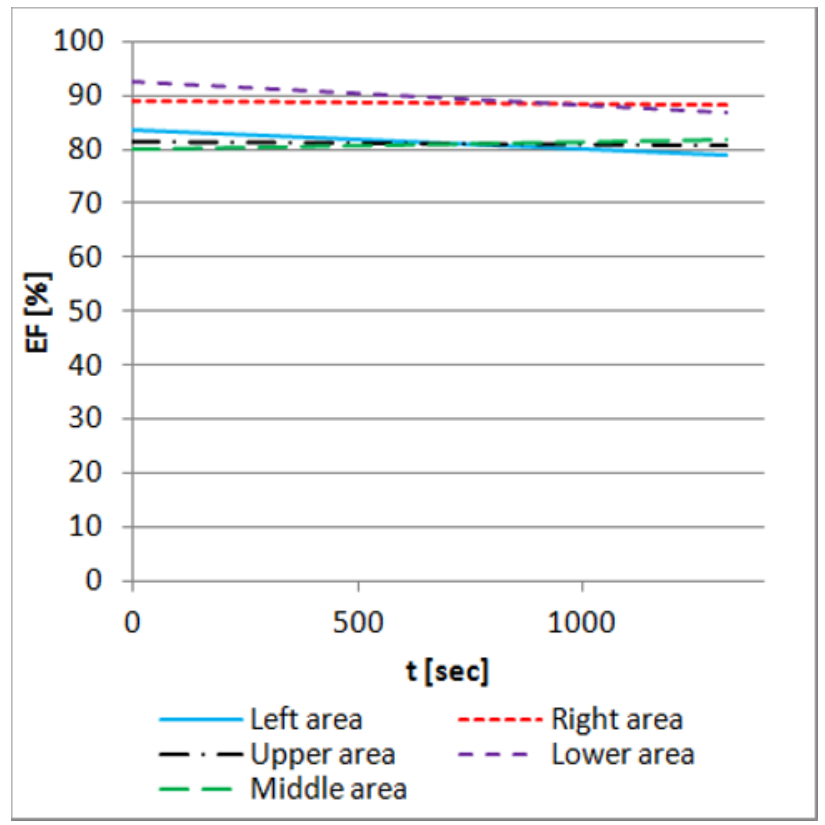

Figure 10. Graph shows the filtration efficiency in different positions versus time.
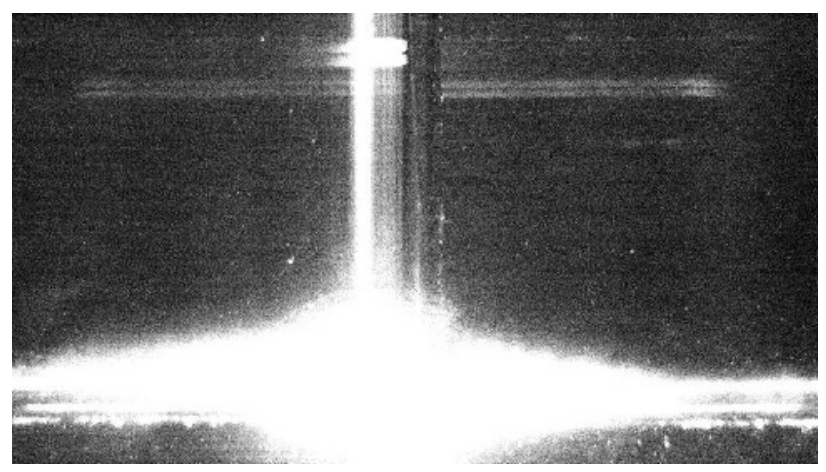

Figure 11. Excessive light reflection of the laser light from glass pipe.

Another factor can be turbulent flow behind the filtration material. Fortunately, the turbulent flow was not proven. Measurements infer not wholly flat velocity profile, but without any huge turbulent structures and recirculation. Next factor can be an inhomogeneity of a concentration of the seeding particles in front of the filter. Seeding particles are much heavier than water and the concentration can be higher in lower part of a pipe than in higher. Water flow during the tests was set on 1 liter per minute and it is speed low enough for sedimentation of the particles.

The measurement results of filtration efficiency were verified by Zeta-sizer ZEN3600 which was used as a simple spectrometer. Two samples of water were taken in front of the filter and behind it during themeasurement. Concentration of the first sample was $500 \mu \mathrm{g} / 1 \mathrm{SiO}_{2}$ and this sample was several times diluted and analyzed by Zeta-sizer. A regression curve was created and a concentration of the second sample was calculated according to the equation in figure 12 .

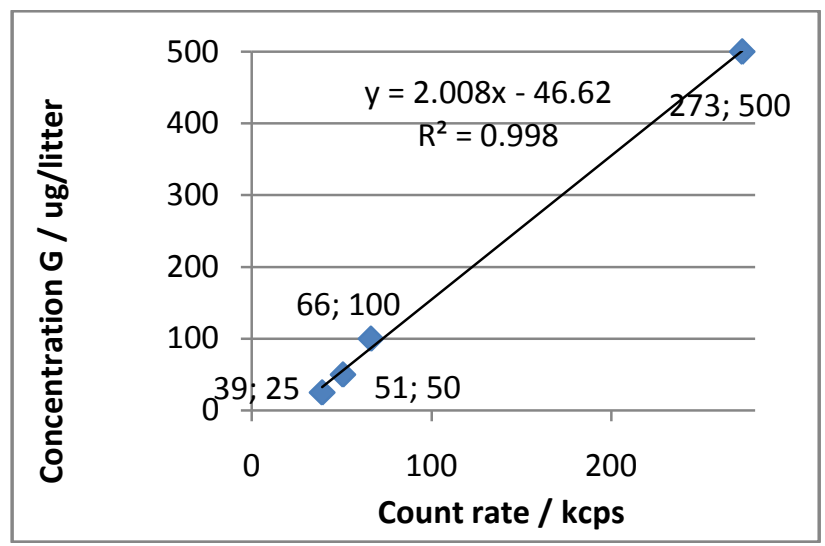

Figure 12. Results from Zeta-sizer. Scattered light intensity versusthe concentration.

A comparison of values of the efficiency is shown in table 6. Mean filtration efficiency $84.0 \%$ was calculated on the basis of visualization of a filtration process. Filtration efficiency calculated by the Zeta-sizer is $92.7 \%$.

To the future, it is important to ensure cleaner water, higher flow rate and more accurate measurement of particle weight. Round pipe where the tested filtration material is mounted is not ideal due to light reflections.

Table 6. Verification of the filtration efficiency by Zeta-sizer.

\begin{tabular}{|l|l|}
\hline Our method: Visualization of flow & $84.0 \%$ \\
\hline Verification by: Zeta-sizer & $92.7 \%$ \\
\hline
\end{tabular}

\section{Conclusion}

The first goal of the performed experiments was selection of the best image analysis method for calculation of the filtration efficiency. We use two methods in the article analysis of the mean grey value and the number of pixels from a histogram. Both methods are sufficient for our experiment. The second image analysis method is better because of its higher R-squared value than in the previous case.

Next goal was a measuring of the filtration efficiency in volume thanks to use of the traverse mechanism. Nonwoven filtration textiles have not absolutely uniform structure as screens. The filtration efficiency of an ideal filtration material is the same in each point of its active surface. It is important to measure uniformity of filtration especially in case of the nanofibrous materials. Uniformity of a filtration depends on mechanical properties of a textile as uniformity of fibres, thickness of a layer and uniformity of a fibre deposition. In other words, the uniformity of the filtration efficiency is another indicator of a quality of the filtration material. This method can also find out some manufacturing defects in a filtration material. 
A tested filter can be investigated in its whole surface. If the number of evaluative areas is large enough, it is possible to achieve enough resolution for diagnosisof a quality of the tested textile. Used measuring method investigates filtration material in process by artificial seeding particles.

\section{Acknowledgments}

This work was supported by the Ministry of Education of the Czech Republic within the project no. 7822 of the Technical University in Liberec.

\section{References}

1. A. Rushton, A. S. Ward, R. G. Holdich, VCH Verlagsgesellschaft mbH, ISBN: 3-527-28613-6 (1996)

2. D. M. Luzhansky, INTC Conference, Donaldson Comapny Inc, p. 7 (2003)

3. M. I. Mishchenko, L. D. Travis, A. A. Lacis, NASA Goddard institute for space studies, ISBN: 0-52178252-X, p. 488 (2004)

4. P. D. Hiscocks, P. Eng, Syscomp electronic design limited, p. 25 (2011)

5. F. Dierks, Basler Vision Technologies, p. 36 (2004) 